\title{
Renormalized energies of superfluorescent bursts from an electron-hole magnetoplasma with high gain in $\operatorname{In}_{x} \mathrm{Ga}_{1-x}$ As quantum wells
}

\author{
J.-H. Kim, ${ }^{1,{ }^{*}}$ J. Lee,${ }^{2, *}$ G. T. Noe, ${ }^{1}$ Y. Wang, ${ }^{3}$ A. K. Wójcik,${ }^{3}$ S. A. McGill, ${ }^{4}$ D. H. Reitze,,${ }^{2, \dagger}$ A. A. Belyanin, ${ }^{3}$ and J. Kono ${ }^{1,5, \ddagger}$ \\ ${ }^{1}$ Department of Electrical and Computer Engineering, Rice University, Houston, Texas 77005, USA \\ ${ }^{2}$ Department of Physics, University of Florida, Gainesville, Florida 32611, USA \\ ${ }^{3}$ Department of Physics, Texas A\&M University, College Station, Texas 77843, USA \\ ${ }^{4}$ National High Magnetic Field Laboratory, Tallahassee, Florida 32310, USA \\ ${ }^{5}$ Department of Physics and Astronomy, Rice University, Houston, Texas 77005, USA
}

(Received 23 May 2012; revised manuscript received 25 November 2012; published 8 January 2013)

\begin{abstract}
We study light emission properties of a population-inverted 2D electron-hole plasma in a quantizing magnetic field. We observe a series of superfluorescent (SF) bursts, discrete both in time and energy, corresponding to the cooperative recombination of electron-hole pairs from different Landau levels. Emission energies exhibit strong renormalization due to many-body interactions among the photogenerated carriers, showing pronounced red shifts as large as $20 \mathrm{meV}$ at $15 \mathrm{~T}$. However, the lowest Landau level emission line remains stable against renormalization and show excitonic magnetic field dependence. Interestingly, our time-resolved measurements show that this lowest-energy SF burst occurs only after most upper states become empty, suggesting that this excitonic stability is related to the "hidden symmetry" of 2D magnetoexcitons expected in the magnetic quantum limit.
\end{abstract}

DOI: 10.1103/PhysRevB.87.045304

PACS number(s): 78.67.De, 78.20.Ls, 78.55.Cr

Optically created electron-hole (e-h) pairs in semiconductors provide a rich system for the study of carrier interaction effects in a highly controllable manner. ${ }^{1-3}$ With continuously tunable pair density, temperature, and magnetic field, one can systematically examine spectral features of bound and unbound carriers in different regimes. In the regimes of high densities and/or low temperatures, a variety of many-body ground states can exist, ${ }^{4-7}$ including Bose-Einstein condensates, e-h droplets, and excitonic insulators and crystals. However, excitons are stable only in the dilute limit, where their Bohr radius is much smaller than the interexciton distance. As the latter approaches the former, a Mott transition ${ }^{8}$ is expected to occur, transforming the insulating excitonic gas into a metallic e-h plasma. In addition, carrier interactions are expected to induce exciton screening and ionization, band-gap renormalization (BGR), and biexciton formation. Furthermore, optical gain develops, and coherent and cooperative processes such as superradiance (SR) and superfluorescence $(\mathrm{SF})^{9-12}$ can dominate the emission spectra.

Here we studied light emission from highly photoexcited undoped InGaAs quantum wells (QWs) at low temperatures and high magnetic fields. Using intense femtosecond laser pulses, we excited e-h pairs at ultrahigh densities and observed bright and sharp emission lines. ${ }^{13,14}$ At the highest field and excitation ranges, strong Landau quantization and Fermi degeneracy increased the density of states and suppressed decoherence, leading to the emergence of intense and coherent pulses of light, or SF bursts, as we recently reported. ${ }^{15}$ In the present study, we examined the influence of many-body interactions on the light emission processes of e-h pairs in the presence of gain, through a detailed magnetic field $(B)$ dependent study of the energies of the SF lines. At zero field, the exciton binding energy approaches zero as the e-h pair density increases, i.e., an excitonic Mott transition. At finite magnetic fields, many intense emission lines appear due to Landau quantization and exhibit pronounced red shifts
( $\gtrsim 20 \mathrm{meV}$ at $15 \mathrm{~T}$ ) from the unperturbed energies determined by linear magnetoabsorption spectroscopy. However, the $B$ dependence of the emission line associated with the lowestenergy [or (00)] Landau level remains excitonic. At the same time, our time-resolved photoluminescence measurements indicate that the (00) SF burst occurs after most of the higher-energy SF bursts occur. Therefore, we interpret this intriguing stability of the excitonic nature of the (00) SF emission in terms of "hidden symmetry" of 2D e-h systems in a strong perpendicular $B$. Namely, theoretical studies have proven that there exists a hidden symmetry ${ }^{16-20}$ for $v_{e}, v_{h} \leqslant 2$ (where $v_{e}, v_{h}$ are the electron and hole filling factors), leading to an exact cancellation of all direct and exchange Coulomb interactions among excitons. This is a consequence of the charge-symmetric nature of e-h Coulomb interactions, and, as a result, the emission energies are independent of interactions in the high- $B$ limit (Fig. 1).

All measurements were performed in the Fast Optics Facility of the National High Magnetic Field Laboratory in Tallahassee, Florida. An undoped multiple quantum well (QW) structure, consisting of fifteen 8-nm $\mathrm{In}_{0.2} \mathrm{Ga}_{0.8} \mathrm{As}$ QWs and 15-nm GaAs barriers, was placed in a 17.5-T superconducting magnet at $5 \mathrm{~K}$. The Mott transition for our sample (effective Bohr radius $a_{\mathrm{B}}^{*} \sim 10 \mathrm{~nm}$ ) is expected to occur at a density of $\left(a_{\mathrm{B}}^{*}\right)^{-2} \sim 10^{12} \mathrm{~cm}^{-2}$. Photoluminescence (PL) and transmission spectra were collected through 0.6-mm-core-diameter multimode optical fibers placed at the center and edge of the sample. A right angle microprism $\left(1 \mathrm{~mm}^{2}\right.$ area $)$ redirected the in-plane emission from the edge of the sample to the fiber. ${ }^{13-15}$ A quartztungsten-halogen lamp was used for white-light transmission measurements. For low and high excitation PL studies, a 25-mW He-Ne laser and an amplified Ti:sapphire laser were used, respectively. The Ti:sapphire source produced pulses centered at $800 \mathrm{~nm}$ with a pulse width of $150 \mathrm{fs}$ at a $1-\mathrm{kHz}$ repetition rate. Pulse energies up to $20 \mu \mathrm{J}$ were used. When 


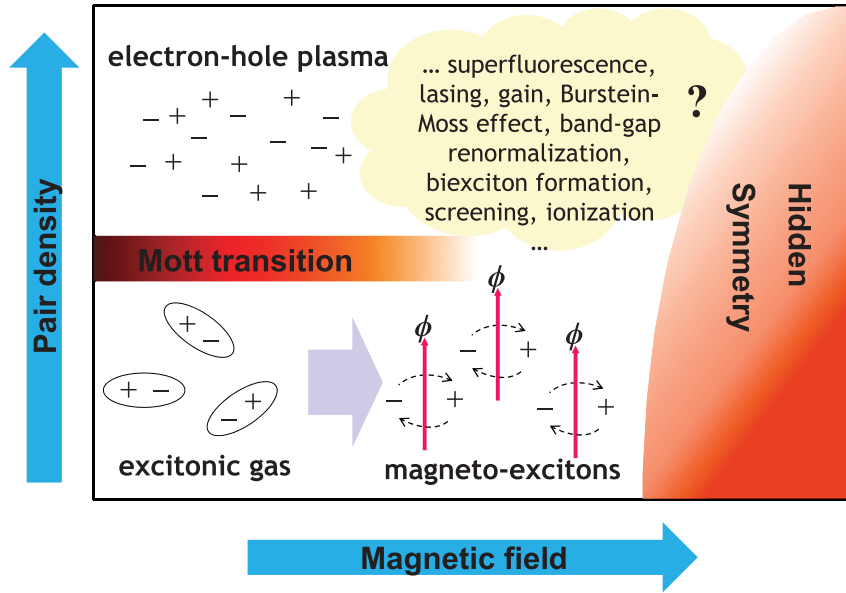

FIG. 1. (Color online) Different phases of electron-hole (e-h) pairs in a charge-neutral semiconductor quantum well as a function of pair density and magnetic field. At zero field, an insulating excitonic gas transforms into a metallic e-h plasma via a Mott transition. In the high-magnetic-field limit, "hidden symmetry"16-18 is expected to prevent excitons from dissociation into free e-h pairs.

focused to a $0.5-\mathrm{mm}$ diameter at the sample, pulse fluences of $10 \mathrm{~mJ} / \mathrm{cm}^{2}$ were obtained, generating e-h pair densities approaching $10^{14} \mathrm{~cm}^{-2}$. Time-resolved PL measurements were performed using a streak camera, and details of the setup are fully described in Ref. 15. All measurements were performed in the Faraday geometry, i.e., the $B$ was perpendicular to the QW plane and parallel to the light propagation direction.

Figures 2(a) and 2(b) show PL and absorption spectra, respectively, taken under weak excitation conditions at different $B$. At $B=0$, the PL spectrum consists of a single peak

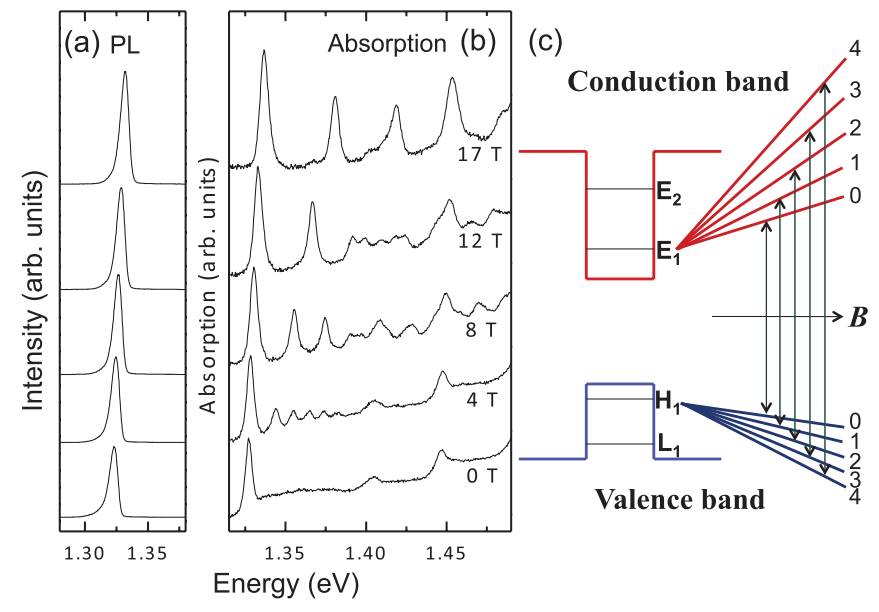

FIG. 2. (Color online) Evolution of emission and absorption spectra of InGaAs quantum wells with increasing magnetic field $(B)$ in the low-density limit. (a) Emission spectra under weak $\mathrm{He}-\mathrm{Ne}$ laser excitation, showing a single peak with a slight diamagnetic shift with $B$. (b) Linear absorption spectra taken with a quartz-tungstenhalogen lamp. The zero-field spectrum consists of a series of step functions superimposed by excitonic peaks at the singularities. (c) Schematic energy level diagram with allowed interband magnetooptical transitions between the $E_{1}$ and $H_{1}$ subbands indicated by arrows. from the band-edge exciton, whereas the absorption spectrum consists of a series of step functions typical of a quasi-2D system, superimposed with excitonic peaks at the singularities. PL shows a single peak at all fields, as shown in Fig. 2(a), exhibiting a characteristic diamagnetic shift $\left(\propto B^{2}\right)$. At finite $B$, the absorption spectrum splits into a series of peaks due to Landau quantization of the conduction and valence bands. In this study, we consider only states within the first electron and heavy-hole subband $\left(E_{1} H_{1}\right)$. We employ the high-field Landau notation $(N, M)$, where $N, M=0,1,2, \ldots$, to specify each $2 \mathrm{D}$ e-h state in a $B$, as opposed to the low-field excitonic notation $(n, m)$; the correspondence between the two is given by $n=\max (N, M)+1$ and $m=N-M \cdot{ }^{21}$ Only states with $N=M$ [or, equivalently, $s$-like $(m=0)$ states] are optically active.

Figures 3(a)-3(d) show time-resolved PL spectra with high excitation using the amplified Ti:sapphire laser, taken with a streak camera at magnetic fields of (a) $17.5 \mathrm{~T}$, (b) $14 \mathrm{~T}$, (c) $10 \mathrm{~T}$, and (d) $6 \mathrm{~T}$. Each $(N, N)$ recombination is observed as a peak both in energy and time, characteristic of a delayed pulse, or burst, of SF. ${ }^{15}$ The number of peaks increases with decreasing $B$, and at each $B$ the (00) burst occurs last. The energies of the observed $(N, N)$ transitions are plotted in Fig. 4 for linear absorption and weak CW excitation PL [in Fig. 4(a)] and SF pulses [in Fig. 4(b)]. The $y$ intercepts at $B=0$ for absorption in Fig. 4(a) provide the ground and excited excitonic energies with a binding energy of $9.83 \mathrm{meV}$ and band gap of $1.338 \mathrm{eV}$. The $\sim 7-\mathrm{meV}$ Stokes shift of the PL with respect to the (00) absorption in Fig. 4(a) is likely due to alloy disorder in the sample. All $N \neq 0 \mathrm{SF}$ transitions, e.g., (11), (22), (33), and (44), shown in Fig. 4(b), are seen to be strongly red-shifted ( $\gtrsim 20 \mathrm{meV}$ at $15 \mathrm{~T}$ ) from the energies of linear magnetoabsorption transitions in Fig. 4(a). In addition, the slope versus $B$ of each of these transitions is reduced, indicating that the effective mass is also renormalized, i.e., enhanced, due to the presence of a large density of e-h pairs. The fit shown in Fig. 4(b) corresponds to electron masses $m_{e}=\{0.0666,0.0689,0.0709,0.0734,0.0754,0.0769\} m_{0}$ for the (11), (22), (33), (44), (55), and (66) transitions, respectively. Furthermore, all SF transitions converge to a single point at zero field, indicating a vanishing exciton binding energy at $B=0$, highlighting the fact that the density is above the Mott density, i.e., excitons are unstable.

The $B$ dependence of the energies of the linear absorption peaks can be calculated by solving the Schrödinger equation for an undoped InGaAs QW with an electron and a heavy hole. The Hamiltonian includes their QW confinement in the growth direction, their interaction with $B$, and the Coulomb interaction between the electron and hole. A general eigenstate of the Hamiltonian includes mixing among different levels in the growth direction. However, for the first few eigenstates of the Hamiltonian, this type of mixing is very small, due to the large energy spacing between them. Thus, we can assume that both the electron and hole are in their ground states described by wave functions $\phi_{e 1}\left(z_{e}\right)$ and $\phi_{h 1}\left(z_{h}\right)$ in the growth direction, and their in-plane motion experiences an effective Coulomb interaction given by ${ }^{22}$

$$
V_{\mathrm{eff}}(\rho)=-\frac{e^{2}}{\epsilon} \int d z_{e} \int d z_{h} \frac{\left|\phi_{e 1}\left(z_{e}\right)\right|^{2}\left|\phi_{h 1}\left(z_{h}\right)\right|^{2}}{\sqrt{\rho^{2}+\left(z_{e}-z_{h}\right)^{2}}},
$$




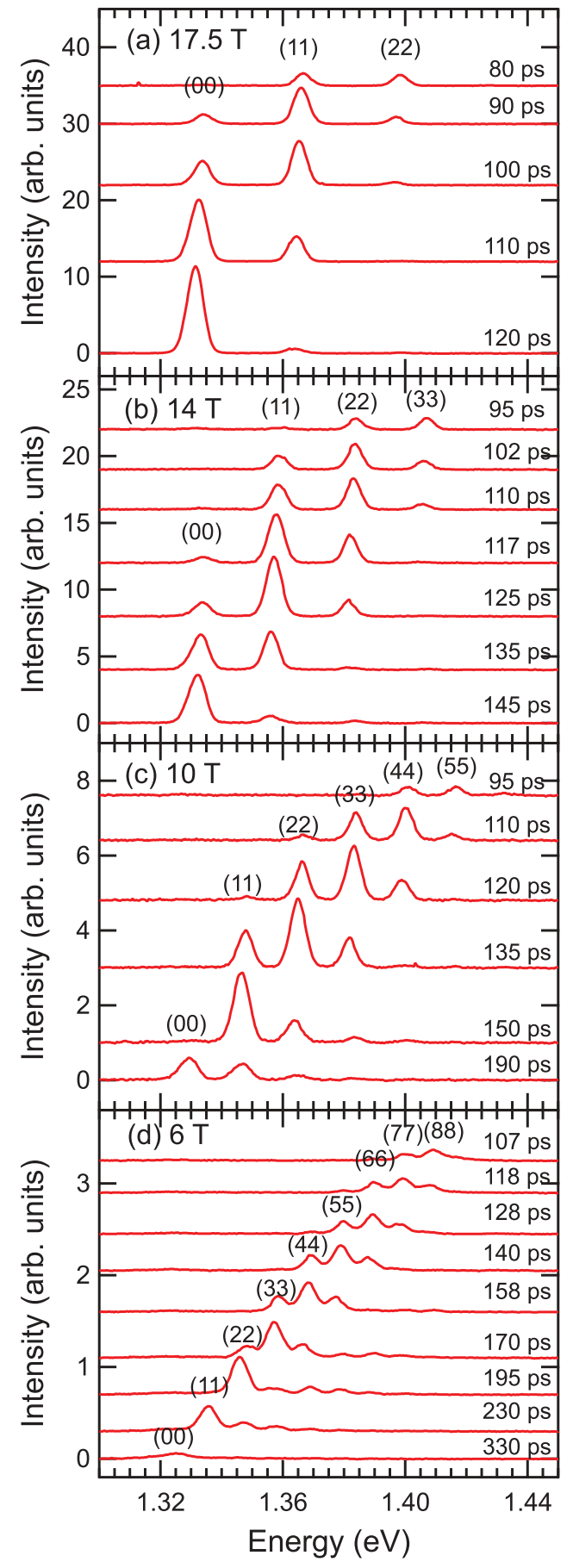

FIG. 3. (Color online) Time-resolved emission spectra at (a) $17.5 \mathrm{~T}$, (b) $14 \mathrm{~T}$, (c) $10 \mathrm{~T}$, and (d) $6 \mathrm{~T}$. Each $(N, N)$ recombination is observed as a delayed pulse, or burst, of superfluorescence. ${ }^{15}$ At a fixed $B$, the delay is longer for smaller $N$; the $N=0$ state is the last to burst.

where $\rho=\left|\vec{\rho}_{e}-\vec{\rho}_{h}\right|$ is the in-plane e-h separation.

Using the symmetric gauge $\vec{A}_{e(h)}=\vec{B} \times \vec{\rho}_{e(h)} / 2$, we transform the in-plane e-h wave function $\Psi\left(\vec{\rho}_{e}, \vec{\rho}_{h}\right)$ as $U(\vec{\rho})=\exp \left[-i(e / 2 c \hbar) \vec{B} \cdot\left(\vec{\rho}_{\mathrm{cm}} \times \vec{\rho}\right)\right] \Psi\left(\vec{\rho}_{e}, \vec{\rho}_{h}\right),{ }^{23}$ where we have set the center-of-mass momentum to be zero since only these states are relevant to optical transitions. If one writes $U(\vec{\rho})=R_{m}(\rho) e^{i m \phi} / \sqrt{2 \pi}$, where $m$ is the angular momentum
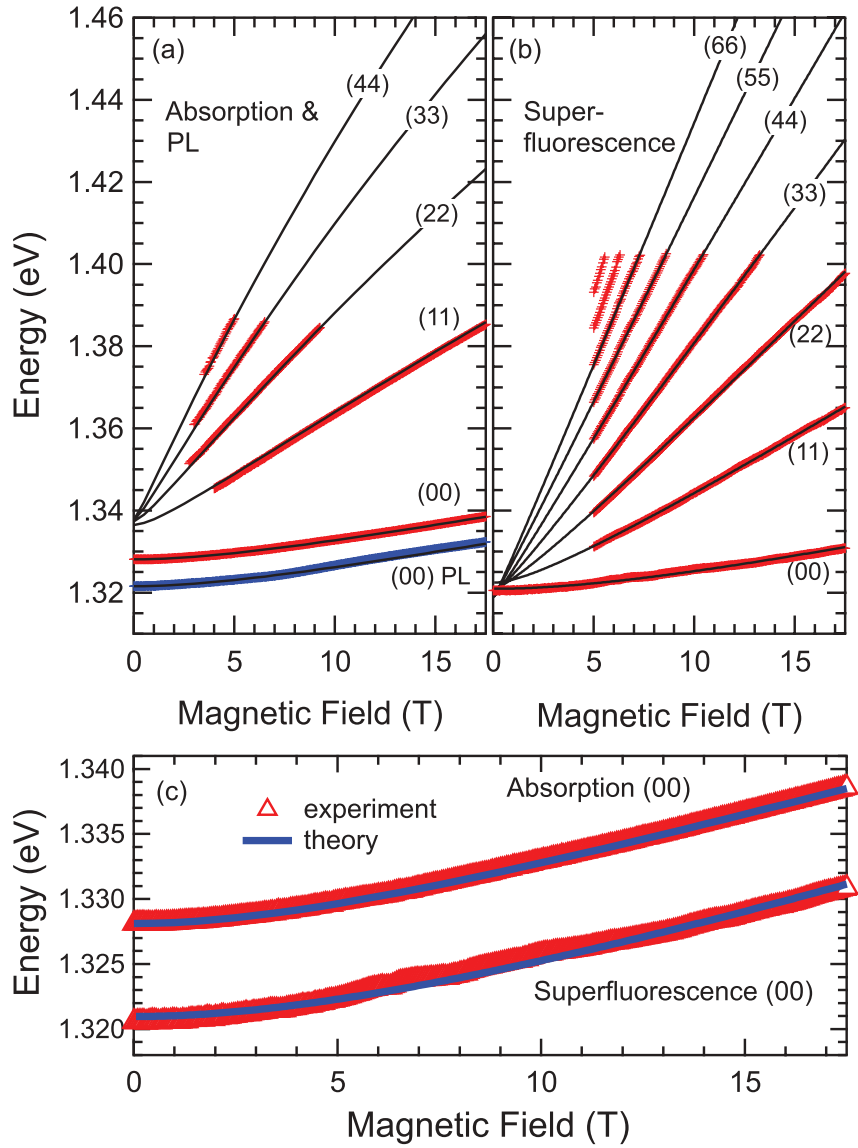

FIG. 4. (Color online) Magnetic field ( $B$ ) dependence of transition energies of observed absorption and emission peaks. (a) Lowintensity white light absorption (red) and photoluminescence (blue) transition energies versus $B$, together with fits using calculations (solid lines) described in the text. The Landau indices $(N, M)$ of each transition are indicated. The $y$ intercepts at zero field provide the ground and excited excitonic energies with a binding energy of $9.83 \mathrm{meV}$ and band gap of $1.338 \mathrm{eV}$. (b) Transition energies of superfluorescent bursts under high excitation versus $B$, together with calculations (solid lines). It is seen that all transitions converge to a single point at zero field, indicating a vanishing exciton binding energy at $B=0$. All transition energies for $(N, N)(N \neq 0)$ are renormalized with respect to those in (a). However, the $B$ dependence of the (00) peak retains its excitonic character. (c) Expanded view of the energy of the (00) line versus $B$ for both absorption and superfluorescence.

number, $R_{m}(\rho)$ satisfies the equation

$$
\begin{gathered}
\left\{-\frac{\hbar^{2}}{2 \mu}\left[\frac{1}{\rho} \frac{d}{d \rho}\left(\rho \frac{d}{d \rho}\right)-\frac{m^{2}}{\rho^{2}}\right]+V_{\mathrm{eff}}(\rho)+\frac{\mu \omega_{c}^{2} \rho^{2}}{8}\right. \\
\left.-\frac{m_{e}-m_{h}}{m_{e}+m_{h}} \frac{m \hbar \omega_{c}}{2}\right\} R_{m}(\rho)=\left(E_{m}-E_{\mathrm{g}, \mathrm{eff}}\right) R_{m}(\rho),
\end{gathered}
$$

where $\mu$ is the reduced mass, $\omega_{\mathrm{c}}=e B / \mu c$, and $E_{\mathrm{g}, \text { eff }}$ is the effective band gap, which includes the band gap of InGaAs and the ground-level confinement energies of the electron and hole. The boundary conditions are that $R_{m}(0)$ is finite and $R_{m}(\infty)=0$. This equation has the Sturm-Liouville form but is singular at $\rho=0$ if $m \neq 0$. It can be solved numerically by using the SLEIGN2 code. ${ }^{24}$ However, it is not easy to include 
nonparabolicity in this code, so we directly integrated Eq. (2) instead. For optical transitions $(m=0)$, there is no singularity, and direct integration is straightforward. The nonparabolicity of both electron and heavy hole can be described by $E(k)=a k^{2}+b k^{4}$, so the reduced mass can be written as $\mu\left(E_{k}\right)=\mu(0)\left(1+\alpha E_{k}\right)$, where $E_{k}$ is the kinetic energy and $\alpha$ is a coefficient. ${ }^{25}$ In the case when the carriers are in a magnetic field and interact with the Coulomb potential, the eigenstates are not plane waves and the nonparabolicity formula for the kinetic energy cannot be applied directly. As a simplified treatment, we used the averaged kinetic energy, which is the eigenenergy of Eq. (2) subtracted by the Coulomb potential averaged over that eigenstate. The energies of the absorption peaks calculated in this way agree with the experimental data extremely well, with deviation less than $1 \mathrm{meV}$, as seen in Figs. 4(a) and 4(c). We used the same set of parameters to calculate the $B$ dependence for all Landau levels: the dielectric constant $\epsilon=12.5, \mu\left(E_{\mathrm{k}}=0\right)=0.043 m_{0}$, where $m_{0}$ is the free electron mass, $\alpha=2.2 \mathrm{eV}^{-1}$, and $E_{\mathrm{g}, \text { eff }}=1.338 \mathrm{eV}$. The calculated exciton binding energy $E_{b}$ at $B=0$ is $9.83 \mathrm{meV}$.

For the SF emission data at high carrier densities, shown in Fig. 4(b), one would expect that the emission spectrum is described in the model of an electron-hole magnetoplasma since excitons are completely ionized at $B=0$. Strikingly, however, the position of the (00) peak still exhibits a clear $B^{2}$ excitonic behavior, as opposed to a linear $B$ dependence indicative of an ionized e-h magnetoplasma. The $B$ dependence of the (00) peak agrees with our solution for a magnetoexciton PL from a noninteracting gas of excitons for the same set of parameters as in the previous paragraph [see Fig. 4(c)].

To interpret these results, we first note the fact that the SF burst from the lowest-energy level, (00), occurs only after most of the upper states become significantly depopulated by their own bursts of emission, as shown in Figs. 3(a)-3(d). Thus, this amazing stability of the excitonic nature of emission at high densities is qualitatively consistent with the concept of the hidden symmetry predicted for $2 \mathrm{D}$ magnetoexcitons in the high- $B$ limit, ${ }^{16,17}$ in which all interexciton interactions are expected to exactly cancel, thereby immunizing their emission lines against shifting or broadening. This is a consequence of the charge-symmetric nature of e-h Coulomb interactions ${ }^{16}$ and has been shown to play an important role in the shake-up process of the lowest Landau level in modulation-doped QWs in high $B .{ }^{19,20}$ However, the ranges of $B$ and density in which stability is observed here go beyond the ranges where hidden symmetry has been shown to be valid. Specifically, hidden symmetry is expected to be valid only in the high- $B$ limit (filling factor $\ll 1$ ), where all inter-Landau-level mixing can be ignored, whereas in our studies many higher Landau levels are close in energy to the (00) state and still carry some population by the time of the SF burst.

The difference of our experiment from previous studies ${ }^{26,27}$ is that we observe stimulated emission from a high-gain system, instead of spontaneous emission. In this case, the peak in the stimulated emission spectrum corresponds to the wave numbers $k$ at which the gain (imaginary part of the frequency $\operatorname{Im}[\omega(k)]$ of unstable electromagnetic modes) has a maximum. The remarkable single-exciton behavior of the emission spectrum is a manifestation of the fact that the gain can still be described by the Elliott-type formula, ${ }^{2,3}$ even at high e-h densities $\left(\sim 10^{12} \mathrm{~cm}^{-2}\right)$ corresponding to a completely filled (00) level. Namely the gain is dramatically enhanced when the recombining electron and hole spatially overlap, forming a neutral two-particle exciton state. Even though the initial spontaneous emission is blue-shifted and spread over a broader range of energies corresponding to the transitions between many-particle states of the system, the stimulated emission is concentrated within a narrow spectral region near the peak of the gain at the single-exciton energy. Previous studies $^{20}$ showed no trace of excitonic features in the spontaneous PL spectra until the filling factor became smaller than 2 .

In conclusion, we studied the energies of superfluorescent bursts from highly photoexcited undoped InGaAs quantum wells at low temperatures and high magnetic fields. We observe strong energy and mass renormalization due to the presence of a large density of electron-hole pairs, as compared to linear magnetoabsorption transitions. At zero field, the exciton binding energy vanishes, indicating that the electron-hole pair density is larger than the Mott density. However, the magnetic-field dependence of the emission line associated with the lowestenergy [or (00)] Landau level remains excitonic. Based on the observation that the $(00)$ burst occurs after most of the higherenergy SF bursts occur, we interpret this stability of the excitonic nature of the (00) SF in terms of hidden symmetry of 2D electron-hole systems in a strong perpendicular magnetic field.

This work was supported by the NSF (through Grants No. DMR-0325474, No. DMR-1006663, No. EEC-0540832, and No. ECCS-0925446). A portion of this work was performed at the National High Magnetic Field Laboratory, supported by NSF Cooperative Agreement No. DMR-0084173 and by the State of Florida. We thank Glenn S. Solomon for providing us with the sample used in this study and L. V. Keldysh for illuminating discussions.
${ }^{*}$ J.-H. Kim and J. Lee equally contributed to this work.

${ }^{\dagger}$ Present Address: LIGO Laboratory, California Institute of Technology, Pasadena, California 91125, USA

${ }^{\ddagger}$ Corresponding author: kono@ rice.edu

${ }^{1}$ H. Haken and S. Nikitine (eds.), Excitons at High Density (SpringerVerlag, Berlin, 1975).

${ }^{2}$ H. Haug and S. W. Koch, Quantum Theory of the Optical and Electronic Properties of Semiconductors (World Scientific, Hackensack, NJ, 1993).
${ }^{3}$ S. W. Koch, M. Kira, G. Khitrova, and H. M. Gibbs, Nat. Mater. 5, 523 (2006).

${ }^{4}$ B. I. Halperin and T. M. Rice, Rev. Mod. Phys. 40, 755 (1968).

${ }^{5}$ P. Nozieres, Physica B + C 117-118, 16 (1983).

${ }^{6}$ L. V. Keldysh, in Bose-Einstein Condensation, edited by A. Griffin, D. W. Snoke, and S. Stringari (Cambridge University Press, Cambridge, 1995), pp. 246-280.

${ }^{7}$ S. A. Moskalenko and D. W. Snoke, Bose-Einstein Condensation of Excitons and Biexcitons: And Coherent Nonlinear 
Optics with Excitons (Cambridge University Press, Cambridge, 2000).

${ }^{8}$ N. F. Mott, Phil. Mag. 6, 287 (1961).

${ }^{9}$ R. H. Dicke, Phys. Rev. 93, 99 (1954).

${ }^{10}$ R. Bonifacio and L. A. Lugiato, Phys. Rev. A 11, 1507 (1975).

${ }^{11}$ A. A. Belyanin, V. V. Kocharovsky, and V. V. Kocharovsky, Quant. Semiclass. Opt. 9, 1 (1997).

${ }^{12}$ A. A. Belyanin, V. V. Kocharovsky, and V. V. Kocharovsky, Quant. Semiclass. Opt. 10, L13 (1998).

${ }^{13}$ Y. D. Jho, X. Wang, J. Kono, D. H. Reitze, X. Wei, A. A. Belyanin, V. V. Kocharovsky, V. V. Kocharovsky, and G. S. Solomon, Phys. Rev. Lett. 96, 237401 (2006).

${ }^{14}$ Y. D. Jho, X. Wang, D. H. Reitze, J. Kono, A. A. Belyanin, V. V. Kocharovsky, V. V. Kocharovsky, and G. S. Solomon, Phys. Rev. B 81, 155314 (2010).

${ }^{15}$ G. T. Noe II, J.-H. Kim, J. Lee, Y. Wang, A. K. Wojcik, S. A. McGill, D. H. Reitze, A. A. Belyanin, and J. Kono, Nat. Phys. 8, 219 (2012).

${ }^{16}$ A. B. Dzyubenko and Y. E. Lozovik, J. Phys. A: Math. Gen. 24, 415 (1991).
${ }^{17}$ V. M. Apal'kov and E. I. Rashba, Pis'ma Zh. Eksp. Teor. Fiz. 53, 420 (1991) [JETP Lett. 53, 442 (1991)].

${ }^{18}$ A. H. MacDonald, E. H. Rezayi, and D. Keller, Phys. Rev. Lett. 68, 1939 (1992).

${ }^{19}$ G. Finkelstein, H. Shtrikman, and I. Bar-Joseph, Phys. Rev. B 56, 10326 (1997).

${ }^{20}$ E. I. Rashba, M. D. Sturge, H. W. Yoon, and L. N. Pfeiffer, Solid State Commun. 114, 593 (2000).

${ }^{21}$ A. H. MacDonald and D. S. Ritchie, Phys. Rev. B 33, 8336 (1986).

${ }^{22}$ E. L. Ivchenko, Optical Spectroscopy of Semiconductor Nanostructures (Alpha Science Intl., Oxford, 2005).

${ }^{23}$ M. Shinada and K. Tanaka, J. Phys. Soc. Jpn. 29, 1258 (1970).

${ }^{24}$ P. B. Bailey, W. N. Everitt, and A. Zettl, ACM Trans. Math. Software 27, 143 (2001).

${ }^{25}$ U. Ekenberg, Phys. Rev. B 40, 7714 (1989).

${ }^{26}$ M. Potemski, J. C. Maan, K. Ploog, and G. Weimann, Solid State Commun. 75, 185 (1990).

${ }^{27}$ L. V. Butov, V. D. Egorov, V. D. Kulakovskii, and T. G. Andersson, Phys. Rev. B 46, 15156 (1992). 